Значение функциональной мультиспиральной компьютерной томографии в диагностике нестабильности позвоночно-двигательных сегментов шейного отдела позвоночника

\author{
Терновой С.К., Серова Н.С., Абрамов А.С., Мискарян Т.И.
}

ФГАОУ ВО «Первый Московский государственный медицинский университет им. И.М. Сеченова» Минздрава России, Институт клинической медицины им. Н.В. Склифосовского, кафедра лучевой диагностики и лучевой терапии, ул. Малая Трубецкая, 8, стр. 2, Москва, 119991, Российская Федерация

Терновой Сергей Константинович, д. М. Н., профессор, академик РАН; orcid.org/0000-0003-4374-1063

Серова Наталья Сергеевна, д. М. н., профессор, чл-коорр. РАН; orcid.org/0000-0001-6697-7824

Абрамов Александр Сергеевич, ассистент кафедры; orcid.org/0000-0002-5739-3170

Мискарян Тамара Ивановна, заведующая учебной частью кафедры

\begin{abstract}
Цель. Нестабильность позвоночно-двигательных сегментов представляет собой весьма актуальную проблему медицины, поскольку имеет весьма серьезные последствия. Сегодня поиск оптимальных методов диагностики продолжается. Нами была предложена новая диагностическая методика - применение функциональной мультиспиральной компьютерной томографии (фМСКТ). Целью работы стало исследование эффективности использования данного метода по сравнению с рентгенографией.

Материал и методы. С помощью новой методики фМСКТ были обследованы 35 пациентов с направительным диагнозом «нестабильность шейного отдела позвоночника», которые ранее прошли рентгенографию шейного отдела позвоночника в прямой и боковой проекциях.

Результаты. Нестабильность позвоночно-двигательных суставов по своим клиническим проявлениям схожа с гипермобильностью шейного отдела позвоночника. По результатам фМСКТ была выполнена оценка встречаемости признаков нестабильности и гипермобильности. При проведении фМСКТ у 48,6\% пациентов выявлена компрессия спинномозгового канала, у 85,7\% обнаружена компрессия корешков спинного мозга. У 11,4\% и 97,1\% больных диагностировано нарушение целостности переднего и заднего опорных комплексов соответственно.

Заключение. Разработанная методика фМСКТ позволяет точно оценить как статику, так и динамику позвоночника и, в отличие от рентгенографии, дает возможность диагностировать вентральные и дорсальные смещения позвонков. Методика рекомендована к широкому применению в клинической практике. Ключевые слова: нестабильность позвоночно-двигательных сегментов; рентгенография; мультиспиральная компьютерная томография; шейный отдел позвоночника.

Конфликт интересов. Авторы заявляют об отсутствии конфликта интересов.

Для цитирования: Терновой С.К., Серова Н.С., Абрамов А.С., Мискарян Т.И. Значение функциональной мультиспиральной компьютерной томографии в диагностике нестабильности позвоночно-двигательных сегментов шейного отдела позвоночника. Вестник рентгенологии и радиологии. 2020; 101(5): $296-303$. https://doi.org/10.20862/0042-4676-2020-101-5-296-303
\end{abstract}

Для корреспонденции: Абрамов Александр Сергеевич, E-mail: 79032324456@ya.ru

\title{
The Value of Functional Multislice Computed Tomography in the Diagnosis of Instability of the Cervical Spinal Motion Segments
}

\section{Sergey K. Ternovoy, Natalya S. Serova, Aleksandr S. Abramov, Tamara I. Miskaryan}

Chair of Radiology and Radiotherapy, Sklifosovskiy Institute for Clinical Medicine, Sechenov First Moscow State Medical University, Ministry of Health of the Russian Federation, ul. Malaya Trubetskaya, 8-2, Moscow, 119991, Russian Federation 
Sergey K. Ternovoy, Dr. Med. Sc., Professor, Academician of RAS; orcid.org/0000-0003-4374-1063

Natalya S. Serova, Dr. Med. Sc., Professor, Corresponding Member of RAS; orcid.org/0000-0001-6697-7824

Aleksandr S. Abramov, Assistant Professor; orcid.org/0000-0002-5739-3170

Tamara I Miskaryan, Head of the Educational Unit

Objective. Instability of the vertebral motion segments is a very urgent problem of medicine, since it has very serious consequences. Today, search for optimal diagnostic methods continues. The authors propose a new diagnostic technique - functional multislice computed tomography (fMSCT). The objective of the study was to investigate the efficiency of the technique versus radiography.

Material and methods. The new technique was used to examine 35 patients with a referral diagnosis of cervical spinal instability, who had previously undergone cervical spine radiography in the frontal and lateral projections.

Results. Instability of the spinal motion joints in its clinical manifestations is similar to the hypermobility of the cervical spine. According to the results of $\mathrm{PMSCT}$, the occurrence of signs of instability and hypermobility was assessed. When performing $\mathrm{fMSCT}$, compression of the spinal canal was detected in $48.6 \%$ of patients, compression of the spinal roots was revealed in $85.7 \%$. Violation of the integrity of the anterior and posterior support complexes was registered in $11.4 \%$ and $97.1 \%$ of patients, respectively.

Conclusion. The developed $\mathrm{fMSCT}$ technique allows to accurately assess both the statics and dynamics of the spine and, unlike radiography, to diagnose ventral and dorsal vertebral displacements. The technique is recommended for widespread use in clinical practice.

Keywords: instability of the spinal motion segments; radiography; multispiral computed tomography; cervical spine.

Conflict of interest. The authors declare no conflict of interest.

For citation: Ternovoy SK, Serova NS, Abramov AS, Miskaryan TI. The value of functional multislice computed tomography in the diagnosis of instability of the cervical spinal motion segments. Journal of Radiology and Nuclear Medicine. 2020; 101(5): 296-303 (in Russian). https://doi.org/10.20862/0042-4676-2020-101-5-296-303 For corresponding: Aleksandr S.Abramov, E-mail: 79032324456@ya.ru

Received June 10, $2020 \quad$ Revised September 22, 2020

Accepted September 23, 2020

\section{Введение}

Нестабильность позвоночно-двигательных сегментов (НПДС) - это вид патологической подвижности позвоночника, которая проявляется в увеличении амплитуды нормальных движений либо возникновении новых атипичных степеней свободы движений. В научных публикациях многие аспекты проблемы НПДС представлены широко и аргументированно, однако выбор оптимальных инструментов для верификации диагноза до сих пор дискутируется [1-3].

Основным методом диагностики НПДС является измерение горизонтального и углового смещения тела шейного позвонка на боковых рентгеновских снимках, однако единый диагностический стандарт для выявления патологии до сих пор отсутствует [4]. Кроме того, из-за наличия отягощающих факторов, таких как морфологическая изменчивость отдельных тел позвонков и вариации рентгенографического увеличения, ошибки измерения также неизбежны $[5,6]$.

Одним из основных рентгенологических тестов является ADI, однако некоторые специалисты указывают на его ненадежность. В частности, C.G. Gajdosik и S. Ostertag отмечают небольшую величину исследуемых показателей, которая может стать причиной неверной интерпретации результатов [7]. По данным систематического обзора, проведенного N. Hutting et al. в 2013 г. и включившего результаты пяти крупных исследований, среди используемых рентгенологических тестов семь обладали достаточной специфичностью, в то время как их чувствительность была не на должном уровне. У предложенных методик сильно варьировались прогнозируемые значения и отношения правдоподобия, в большинстве случаев отмечен большой разброс доверительных интервалов, что свидетельствует об их низкой диагностической ценности [8]. Работы других авторов также свидетельствуют о недостаточной информативности функциональной рентгенографии: в 30-95\% случаев полученные изображения неадекватны, кроме того, высока доля ложноположительных результатов [9-11].

Некоторые исследователи полагают, что компьютерная томография и магнитно-резонансная томография имеют большой потенциал для диагностики НПДС [7]. Однако есть данные, что эти методики не обладают достаточной диагностической ценностью и способствуют пропуску патологии и хронизации процесса [12]. Именно поэтому назрела необходимость разработки высоковалидных методов диагностики данной патологии. 
Цель работы - оценить результативность использования новой методики функциональной мультиспиральной компьютерной томографии (ФМСКТ) у пациентов с НПДС в шейном отделе позвоночника (ШОП).

\section{Материал и методы}

На базе Российско-японского центра визуализации проведено когортное исследование. В него вошли 35 пациентов с направительным диагнозом «нестабильность позвоночно-двигательных сегментов позвоночника». До поступления в наше отделение больные прошли диагностическое обследование в других стационарах, всем им была выполнена рентгенография ШОП.

В гендерном составе преобладали женщины, их было $60 \%(n=21)$; соответственно, численность мужчин составила $40 \%(n=14)$. Средний возраст пациентов составил $46,3 \pm 2,5$ года (от 25 до 65 лет).

При выполнении фМСКТ применялся аппарат Toshiba Aquilion One (TSX301C). При проведении исследования в движении дополнительно осуществлялось построение мультипланарных и 3D-реконструкций. Внедряемый нами метод был ранее запатентован как «Способ функциональной мультиспиральной компьютерно-томографической диагностики нестабильности позвоночно-двигательных сегментов шейного отдела позвоночника» (RU2637829C1 от 15.06.2016) [13].

Статистическая обработка полученных данных была проведена с использованием программ Statistica v.10.0. Поскольку распределение данных было нормальным, для их описания использовались среднее (M) и стандартное отклонение (SD).

\section{Результаты}

Основной жалобой пациентов была головная боль, наблюдавшаяся в 32 наблюдениях $(91,4 \%)$. Помимо локальных симптомов в шее, больных также беспокоили и жалобы общего характера. Более подробно жалобы пациентов представлены на рисунке 1.

В ходе клинического осмотра отмечено наличие патологических изменений, в том числе напряжение шейно-затылочных мышц, болезненность при пальпации в области поперечных и остистых отростков, ограничение движений в ШОП и гиперлордоз. Данные симптомы встречались у подавляющего числа пациентов (рис. 2).
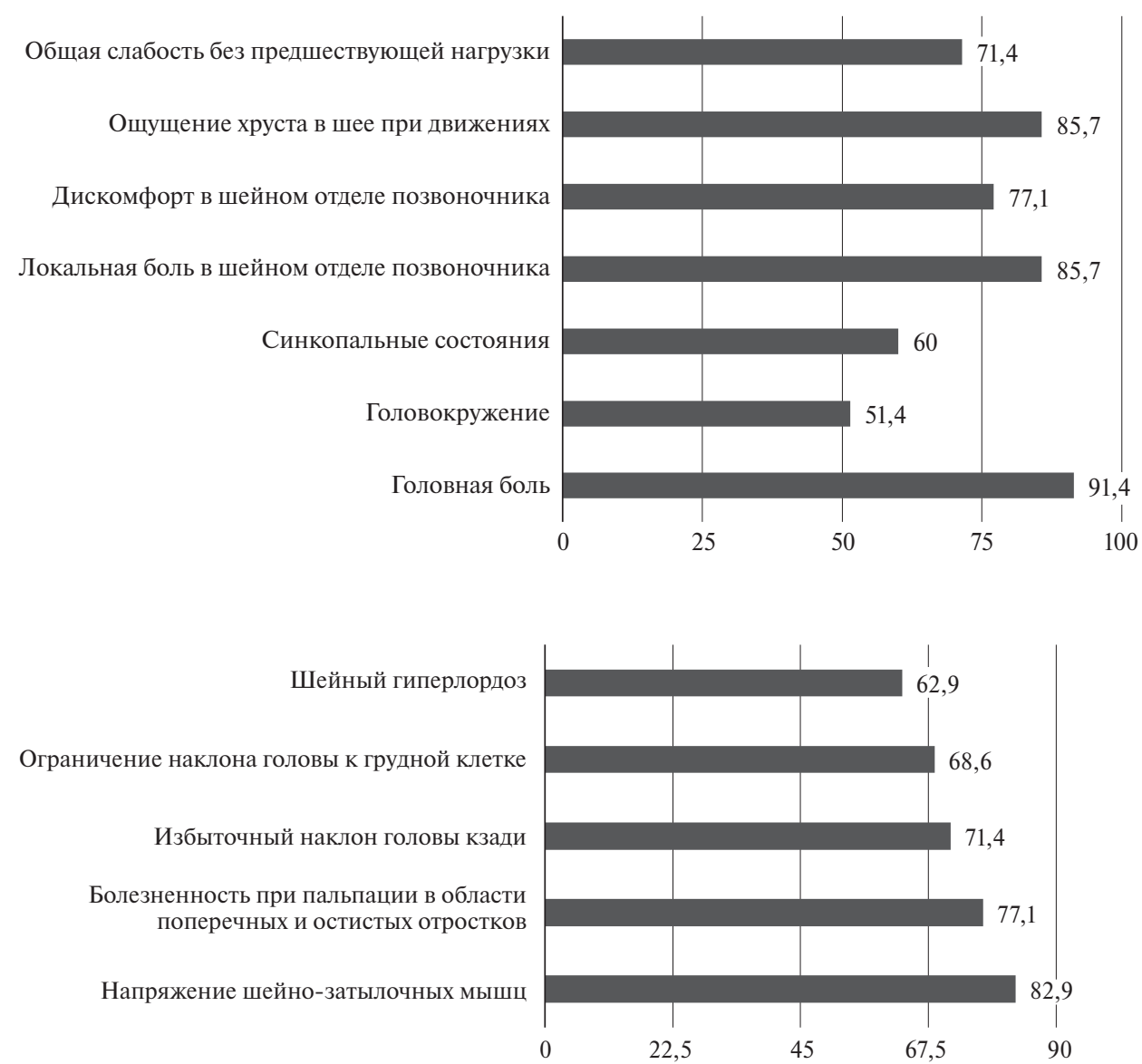

Puc. 1. Встречаемость различных жалоб у исследуемых пациентов (в \%)

Fig. 1. The incidence of different complaints in the studied patients (\%).

General weakness without previous load; Crunching sensation in the neck when moving; Discomfort in the cervical spine; Local pain in the cervical spine; Syncopal states; Dizziness; Headache

Pис. 2. Встречаемость различных клинических симптомов у исследуемых пациентов (в \%)

Fig. 2. The incidence of different clinical symptoms in the studied patients (\%).

Cervical hyperlordosis; Limited flexing of the head to the chest; Excessive posterior tilt of the head; Pain on palpation in the area of transverse and spinous processes; Tension of the neck and occipital muscles 


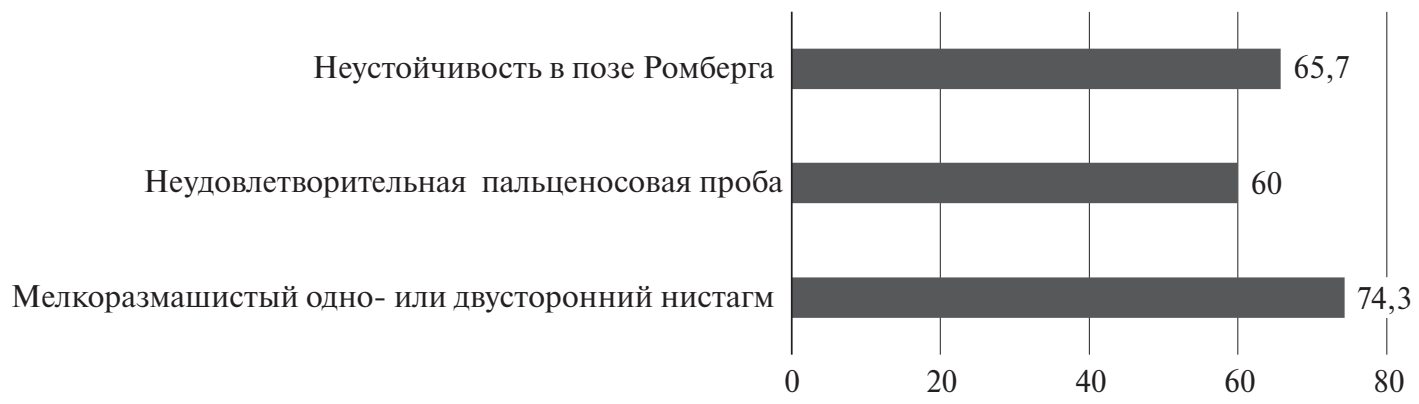

Puc. 3. Встречаемость признаков вертебробазилярной недостаточности у исследуемых пациентов (в \%)

Fig. 3. The incidence of vertebrobasilar insufficiency signs (\%).

Instability in the Romberg test; Unsatisfactory finger-to-nose test; Small-scale single- or double-sided nystagmus

Также у большинства обследуемых наблюдались клинические признаки вертебробазилярной недостаточности (рис. 3).

При выполнении рентгенологического исследования смещение позвонков только при сгибании выявлено у 10 пациентов $(28,6 \%)$, только при разгибании - у 14 (40\%). Признаки НПДС как в положении флексии, так и в положении экстензии отмечены в 10 случаях (28,6\%).

При проведении фМСКТ в положении сгибания у 20 больных $(57,14 \%)$ диагностированы нестабильные ретролистезы, у 14 (40,0\%) выявлены антелистезы. Также в исследовании отмечены пациенты с сочетанием двух видов листеза (5\%) (рис. 4).

При выполнении сгибания у 19 больных $(54,3 \%)$ обнаружено смещение С4-позвонка, средняя амплитуда 2,5 \pm 0,6 мм (1-4 мм). Патологическая мобильность С3-позвонка выявлена у 6 пациентов (30\%), средняя амплитуда при функциональных нагрузках составила 2,5 $\pm 0,7 \mathrm{~mm}$ (1-5 мм). Патологическая подвижность С5-позвонка диагностирована в 4 наблюдениях (20\%), средняя амплитуда $2,5 \pm 0,6$ мм (1-3 мм). Новые патологические степени свободы С2-позвонка наблюдались у 6 пациентов $(17,1 \%)$, средняя амплитуда $2,3 \pm 0,6$ мм (1-4 мм). Смещение С6-позвонка отмечено у 4 больных (11,4\%), средняя амплитуда 2,75 $\pm 0,45$ мм (2-3 мм).

При выполнении разгибания чаще всего встречалось смещение С3- и С4-позвонков. Наличие дополнительной степени свободы С3-позвон- ка при экстензии ШОП установлено у 14 пациентов (40\%), среднее смещение достигало 2,4 \pm 0,6 мм (1-4 мм). Нестабильность С4-позвонка диагностирована у 19 больных (54,2\%), средняя амплитуда смещения 2,4 0,7 мм (1-4 мм). Патологическая подвижность С5-позвонка выявлена в 14 наблюдениях (40\%), средняя амплитуда составила 2,5 0 0,6 мм (1-4 мм). Чуть реже встречалась нестабильность С2-позвонка - у 8 пациентов (22,9\%), средняя амплитуда смещения 2,2 \pm 0,6 мм (1-5 мм). Смещение С6-позвонка в положении максимального разгибания было установлено только в 1 случае (2,8\%), его величина составила 2,25 мм.

В позе максимального сгибания нестабильность двух шейных позвонков диагностирована у 21 пациента (60\%). Реже наблюдалась избыточная мобильность одного или трех позвонков. Так, смещение
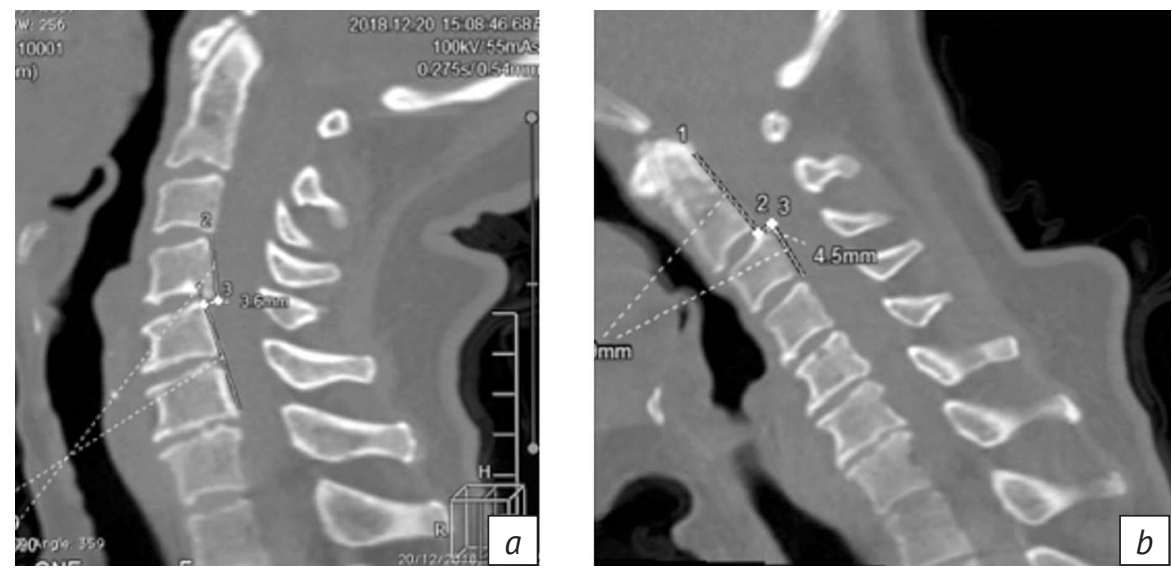

Puc. 4. Пациент М., 46 лет, во время выполнения функциональной пробы:

$a$ - положение максимального разгибания в шейном отделе позвоночника, отмечается смещение тела С5-позвонка кпереди на 3,6 мм;

$b$ - положение максимального сгибания в шейном отделе позвоночника, отсутствует смещение тела С5-позвонка, отмечается ретролистез тела С3-позвонка до 4,5 мм

Fig. 4. Patient M., aged 46, during a functional test:

$a$ - position of maximum extension in the cervical spine; displacement of the C5 vertebra body anteriorly by $3.6 \mathrm{~mm}$;

$b$ - position of maximum flexion in the cervical spine; there is no displacement of the C5 vertebra body; retrolisthesis of the C3 vertebra body up to $4.5 \mathrm{~mm}$ 
Признаки нестабильности и гипермобильности шейного отдела позвоночника у исследуемых пациентов, n (\%)

Signs of cervical spinal instability and hypermobility in the studied patients, $\mathrm{n}(\%)$

\begin{tabular}{l|c}
\hline \multicolumn{1}{c|}{ Признак } & Количество \\
\hline Увеличение амплитуды в шейном отделе позвоночника & $29(82,8)$ \\
Ограничение движений в шейном отделе позвоночника & $28(80,0)$ \\
Сагиттальная трансляция тел шейных позвонков более 3 мм & $31(88,6)$ \\
Угловая деформация* более 11 & $31(88,6)$ \\
Боль в шее & $18(94,3)$ \\
Неврологические расстройства & $29(82,8)$ \\
Напряжение мышц подзатылочной области & $30(85,7)$ \\
\hline
\end{tabular}

* Угол между линиями, проведенными по нижней и верхней замыкательным пластинам тел вышележащего и нижележащего шейных позвонков соответственно.

одного позвонка диагностировано у 8 человек $(22,9 \%)$, трех - у 6 больных $(17,1 \%)$. В позе максимального разгибания смещение одного, двух и трех позвонков встречалось в 14 (40\%), 12 (34,3\%) и 10 (28,6\%) случаях соответственно.

Известно, что НПДС по своим клиническим проявлениям схожа с гипермобильностью шейного отдела позвоночника, которая, в отличие от первой, обусловлена анатомо-физиологическими особенностями. Поэтому по результатам фМСКТ была выполнена оценка встречаемости признаков нестабильности и гипермобильности (см. таблицу).

По итогам проведения рентгенографии у 3 пациентов диагноз НПДС был выставлен неверно. В этих случаях имела место гипермобильность. Таким образом, количество ложноположительных результатов по данным функциональной рентгенографии составило 8,6\%.

При оценке состояния атлантоосевого сустава у 31 пациента $(88,6 \%)$ были выявлены явления субхондрального остеосклероза, свидетельствующие о поздних стадиях остеоартроза, у 23 больных $(65,7 \%)$ отмечалась кистозная перестройка костной ткани, которая чаще всего говорит об обострении патологического процесса. У 1 пациента (2,9\%) был диагностирован артроз унковертебральных сочленений.

У всех больных межпозвонковые суставы имели склерозированные суставные фасетки, заострение тел позвонков наблюдалось в 24 случаях $(68,6 \%)$. У 1 пациента отмечена компрессия дурального мешка. В 12 случаях (34,3\%) регистрировалось выстояние костных остеофитов в полость канала спинного мозга, величина которого варьировала от 2,3 до 3,3 мм.

У 1 пациента наблюдалось обызвествление передней и задней продольных связок на уровне C5-C7. Еще у 2 больных (5,7\%) было зафиксировано обызвествление передней и задней продольных связок на уровне C6-C7.
Несомненным преимуществом фМСКТ является то, что она позволяет видеть динамические изменения позвоночника. В частности, у 3 пациентов $(8,6 \%)$ отмечено возникновение вакуум-эффекта на уровне межпозвонкового диска C4-C5 при выполнении разгибания и его исчезновение в положении максимального сгибания. Статичная рентгенография данные изменения увидеть не позволила.

Нарушение целостности переднего опорного комплекса наблюдалось в 4 случаях $(11,4 \%)$, целостность заднего опорного комплекса была нарушена у 34 пациентов $(97,1 \%)$.

В привычном положении, лежа на спине, смещение позвонков в сагиттальной плоскости было зафиксировано у 1 больного (2,9\%). Смещение позвонков в сагиттальной плоскости при максимальном сгибании отмечено в 11 случаях $(31,4 \%)$, при разгибании - в 8 случаях (22,9\%).

При фМСКТ также есть возможность оценить наличие компрессии спинномозгового канала. В статическом положении она была диагностирована у 7 пациентов (20\%), а при движении - у 17 (48,6\%). У большого числа больных (30 человек - 85,7\%) была выявлена компрессия корешков спинного мозга.

\section{Обсуждение}

Таким образом, сопоставляя результаты применения двух диагностических методов - рентгенографии и фМСКТ, мы видим несомненное преимущество последней. Разработанная методика фМСКТ позволяет точно оценить как статику, так и динамику позвоночника и, в отличие от рентгенографии, дает возможность диагностировать вентральные и дорсальные смещения позвонков. Кроме того, в отличие от рентгенографии, фМСКТ позволяет визуализировать структурные изменения позвонков, грыжи межпозвонковых дисков, вакуум-феномен, стеноз позвоночного канала, изменения в атлантоосевом сочленении, артроз полулунных и суставных отростков, патологию связочного аппарата. 


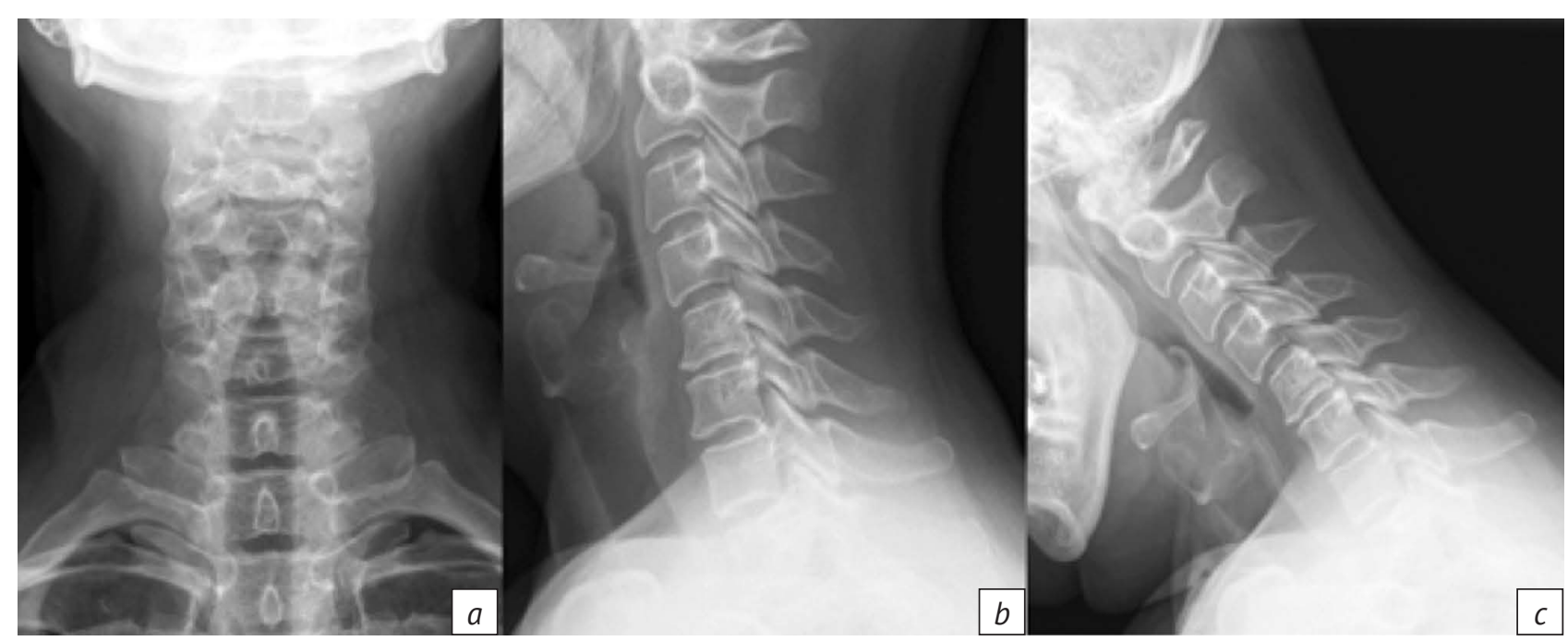

Puc. 5. Пациент Н., 40 лет, стандартная рентгенография с выполнением функциональной пробы на сгибание в положении стоя. Во время выполнения функциональной пробы рентгенологические признаки патологического смещения на уровне С4-C5 (a-c) Fig. 5. Patient N., aged 40 , standard radiography with a functional flexion test performed in a standing position. During a functional test, there are Rg-signs of pathological displacement at C4-C5 level $(a-c)$

Низкая информативность рентгенографии во многом была обусловлена плохим качеством выполненных снимков. Кроме того, повреждения С6, C7 на большинстве рентгенограмм перекрывались тенью надплечий. Еще одной причиной некорректных диагнозов по результатам рентгенографии была неправильная укладка пациентов.

Важным плюсом новой методики является возможность дифференцирования двух состояний - НПДС и гипермобильности шейного отдела позвоночника, которое рентгенологическое исследование провести не позволяет [14]. В нашем исследовании у 3 больных по результатам рентгенографии был выставлен диагноз НПДС. И только проведение МСКТ с функциональной пробой позволило верифицировать диагноз (рис. 5, 6).

Несмотря на кажущуюся схожесть данных состояний, их отождествление неуместно. Гипермобильность представляет собой увеличенный диапазон движений в суставах, вызванный слабостью связок, в то время как нестабильность позвоночника чаще всего возникает в результате травмы или серьезных воспалительных артропатий. НПДС грозит развитием серьезной невро-
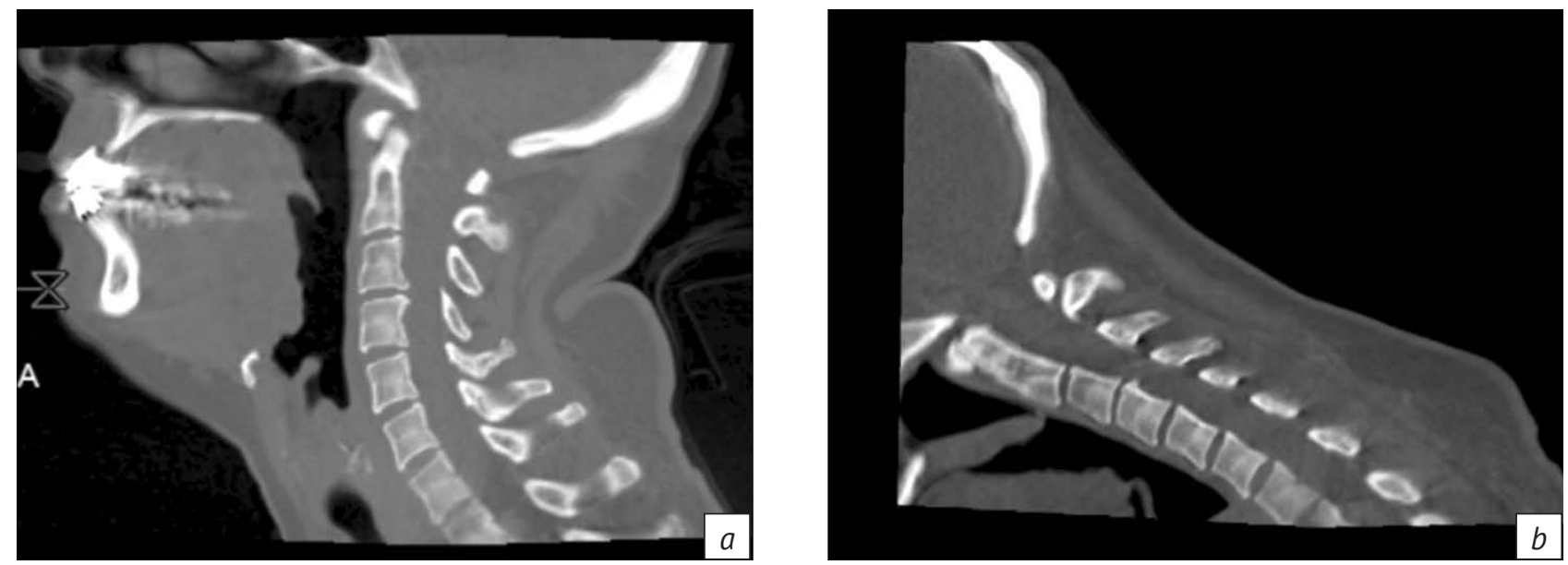

Puc. 6. Пациент Н., 40 лет. Во время выполнения функциональной пробы на сгибание патологического смещения позвонков не выявлено:

$a$ - максимальное разгибание шейного отдела позвоночника;

$b$ - максимальное сгибание шейного отдела позвоночника

Fig. 6. Patient N., aged 40. During the functional tests for flexion, pathological displacement of the vertebrae were not identified: $a$ - maximum extension of the cervical spine;

$b$ - maximum flexion of the cervical spine 
логической патологии с исходом в инвалидность или даже смерть пациента. В таких условиях срочно требуется стабилизация [15]. Гипермобильность может иногда вызывать боль, возникающую из-за пораженных элементов скелета, и может даже приводить к появлению периодических неврологических симптомов, но пораженные люди не подвергаются непосредственному риску внезапного тяжелого неврологического ухудшения [16].
Безусловно, внедрение новой методики требует дальнейших исследований ее диагностических возможностей, а также оценки ее использования в клинической практике.

\section{Заключение}

Внедренный и разработанный нами метод фМСКТ при НПДС шейного отдела позвоночника позволяет повысить точность диагностики и может быть широко внедрен в клиническую практику.

\section{Литература}

1. Kaale BR, Krakenes J, Albrektsen G, Wester K. Clinical assessment techniques for detecting ligament and membrane injuries in the upper cervical spine region: a comparison with MRI results. Man Ther. 2008; 13(5): 397-403. doi: 10.1016/j.math.2007.03.007

2. Hutting NV, Verhagen AP, Vijverman V, Keesenberg MD, Dixon G, Scholten-Peeters GG. Diagnostic accuracy of premanipulative vertebrobasilar insufficiency tests: a systematic review. Man Ther. 2013; 18(3): 177-82. doi: 10.1016/j.math.2012.09.009

3. Кравченко А.И., Климовицький Ф.В. Клинико-рентгенологическая верификация цервикальной нестабильности позвоночника у детей подросткового возраста. Травма. 2015 16(5): $95-8$.

4. Alizada M, Li RR, Hayatullah G. Cervical instability in cervical spondylosis patients: significance of the radiographic index method for evaluation. Orthopade. 2018; 47(12): 977-85. doi: 10.1007/s00132-018-3635-3

5. Волков А.А., Белосельский Н.Н., Прибытков Ю.Н. Рентгеновские признаки дистрофических изменений позвоночного столба в условиях нормальной и сниженной минеральной плотности ткани. Российский электронный журнал лучевой диагностики. 2016; 6(1): 55-64. doi: 10.18411/a-2016-006

6. Абрамов А.С., Терновой С.К., Серова Н.С. Возможности рентгеновских методов диагностики в оценке нестабильности позвоночно-двигательных сегментов шейного отдела позвоночника. Современные проблемы науки и образования. 2019; 3: 184.

7. Gajdosik CG, Ostertag S. Cervical instability and Down syndrome: review of the literature and implications for physical therapists. Pediatr Phys Ther. 1996; 8(1): 31-6.

8. Hutting N, Scholten-Peeters GG, Vijverman V, Keesenberg MD, Verhagen AP. Diagnostic accuracy of upper cervical spine instability tests: a systematic review. Phys Ther. 2013; 93(12): 1686-95. doi: 10.2522/ptj.20130186

9. Nasir S, Hussain M, Mahmud R. Flexion/extension cervical spine views in blunt cervical trauma. Chin J Traumatol. 2012; 15(3): 166-9.

10. Sim V, Bernstein MP, Frangos SG, Wilson CT, Simon RJ, McStay CM, et al. The (f)utility of flexion-extension C-spine films in the setting of trauma. Am J Surg. 2013; 206(6): 929-33. doi: 10.1016/j.amjsurg.2013.09.001

11. Oh JJ, Asha SE. Utility of flexion-extension radiography for the detection of ligamentous cervical spine injury and its current role in the clearance of the cervical spine. Emerg Med Australas. 2016; 28(2): 216-23. doi: 10.1111/1742-6723.12525

12. Щедренок В.В., Захматова Т.В., Могучая О.В., Себелев К.И., Красношлык П.В. Алгоритм хирургического лечения дегене- ративных заболеваний и травмы шейного отдела позвоночника. Medline.ru. Российский биомедицинский журнал. 2014; 15(2): 281-98.

13. Терновой С.К., Серова Н.С., Абрамов А.С., Терновой К.С. Методика функциональной мультиспиральной компьютерной томографии в диагностике нестабильности позвоночно-двигательных сегментов шейного отдела позвоночника. Российский электронный журнал лучевой диагностики. 2016; 6(4): 38-43. doi: 10.21569/2222-7415-2016-6-4-38-43

14. Терновой С.К., Лычагин А.В., Серова Н.С., Абрамов А.С., Черепанов В.Г.Применение функциональной мультиспиральной компьютерной томографии в диагностике нестабильности позвоночно-двигательных сегментов шейного отдела позвоночника. Кафедра травматологии и ортопедии. 2019; 1: 41-7. doi: 10.17238/issn2226-2016.2019.1.41-47

15. Терновой С.К., Лычагин А.В., Серова Н.С., Абрамов А.С., Черепанов В.Г., Мискарян Т.И. Результаты применения функциональной мультиспиральной компьютерной томографии при хирургическом лечении пациентов с нестабильностью шейного отдела позвоночника. Российский электронный журнал лучевой диагностики. 2019; 9(2): 55-62. doi: 10.21569/2222-7 415-2019-9-2-55-62

16. Brodbelt AR, Flint G. Ehlers Danlos, complex Chiari and cranio-cervical fixation: how best should we treat patients with hypermobility? $\mathrm{Br} J$ Neurosurg. 2017; 31(4): 397-8. doi: 10.1080/02688697.2017.1386282

\section{References}

1. Kaale BR, Krakenes J, Albrektsen G, Wester K. Clinical assessment techniques for detecting ligament and membrane injuries in the upper cervical spine region: a comparison with MRI results. Man Ther. 2008; 13(5): 397-403. doi: 10.1016/j.math.2007.03.007

2. Hutting NV, Verhagen AP, Vijverman V, Keesenberg MD, Dixon G, Scholten-Peeters GG. Diagnostic accuracy of premanipulative vertebrobasilar insufficiency tests: a systematic review. Man Ther. 2013; 18(3): 177-82. doi: 10.1016/j.math.2012.09.009

3. Kravchenko OI, Klymovytskyi FV. Clinico-roentgenological verification of cervical instability in adolescents. Trauma. 2015; 16(5): $95-8$ (in Russian).

4. Alizada M, Li RR, Hayatullah G. Cervical instability in cervical spondylosis patients: significance of the radiographic index method for evaluation. Orthopade. 2018; 47(12): 977-85. doi: $10.1007 / \mathrm{s} 00132-018-3635-3$ 
5. Volkov AA, Belosel'sky NN, Pribytkov Yu N. X-ray signs of spinal column distrophic changes within normal and decreased bone mineral density. Russian Electronic Journal of Radiology. 2016; 6(1): 55-64 (in Russian). doi: 10.18411/a-2016-006

6. Abramov AS, Ternovoy SK, Serova NS. Possibilities of the methods of radiation diagnostics in the estimation of cervical spine vertebral-motor segment instability. Modern Problems of Science and Education. 2019; 3: 184 (in Russian).

7. Gajdosik CG, Ostertag S. Cervical instability and Down syndrome: review of the literature and implications for physical therapists. Pediatr Phys Ther. 1996; 8(1): 31-6.

8. Hutting N, Scholten-Peeters GG, Vijverman V, Keesenberg MD, Verhagen AP. Diagnostic accuracy of upper cervical spine instability tests: a systematic review. Phys Ther. 2013; 93(12): 1686-95. doi: 10.2522/ptj.20130186

9. Nasir S, Hussain M, Mahmud R. Flexion/extension cervical spine views in blunt cervical trauma. Chin J Traumatol. 2012; 15(3): 166-9.

10. Sim V, Bernstein MP, Frangos SG, Wilson CT, Simon RJ, McStay CM, et al. The (f)utility of flexion-extension C-spine films in the setting of trauma. Am J Surg. 2013; 206(6): 929-33. doi: 10.1016/j.amjsurg.2013.09.001

11. Oh JJ, Asha SE. Utility of flexion-extension radiography for the detection of ligamentous cervical spine injury and its current role in the clearance of the cervical spine. Emerg Med Australas. 2016; 28(2): 216-23. doi: 10.1111/1742-6723.12525
12. Shchedrenok VV, Zakhmatova TV, Moguchaya OV, Sebelev KI, Krasnoshlyk PV. Algorithm of surgery at degenerative deseases and inyury of cervical department of a backbone. Medline.ru. Russian Biomedical Journal. 2014; 15(2): 281-98 (in Russian).

13. Ternovoy SK, Serova NS, Abramov AS, Ternovoy KS. Functional multislice computed tomography in the diagnosis of cervical spine vertebral-motor segment instability. Russian Electronic Journal of Radiology. 2016; 6(4): 38-43 (in Russian). doi: 10.215 69/2222-7415-2016-6-4-38-43

14. Ternovoy SK, Lychagin AV, Serova NS, Abramov AS, Cherepanov VG. Application of the functional multipyral computer tomography in the diagnostic diagnostics and the retail motor segments of the neck division of the spine. Department of Traumatology and Orthopedics. 2019; 1: 41-7 (in Russian). doi: 10.17238/issn2226-2016.2019.1.41-47

15. Ternovoy SK, Lychagin AV, Serova NS, Abramov AS, Cherepanov VG, Miskaryan TI. The results of the application of functional multispiral computed tomography in the surgical treatment of patients with cervical spine instability. Russian Electronic Journal of Radiology. 2019; 9(2): 55-62 (in Russian). doi: 10.21569/2222-7415-2019-9-2-55-62

16. Brodbelt AR, Flint G. Ehlers Danlos, complex Chiari and cranio-cervical fixation: how best should we treat patients with hypermobility? $\mathrm{Br} J$ Neurosurg. 2017; 31(4): 397-8. doi: 10.1080/02688697.2017.1386282 